\title{
Down-Regulation of Filamin Ainteracting protein 1-like Is Associated with Promoter Methylation and an Invasive Phenotype in Breast, Colon, Lung and Pancreatic Cancers
}

\author{
Mijung Kwon, Soo Jin Lee, Srilakshmi Reddy, Yevangelina Rybak, Asha Adem, Steven K. Libutti ${ }^{*}$ \\ Department of Surgery, Albert Einstein College of Medicine of Yeshiva University, Bronx, New York, United States of America
}

\begin{abstract}
Identifying key mediators of cancer cell invasion and metastasis is critical to the development of more effective cancer therapies. We previously identified Filamin A interacting protein 1-like (FILIP1L) as an important inhibitor of cell migration and invasion in ovarian cancer. FILIP1L expression was inversely correlated with the invasive potential of ovarian cancer cell lines and ovarian cancer specimens. We also demonstrated that DNA methylation in the FILIP1L promoter was a mechanism by which FILIP1L was down-regulated in ovarian cancer. In our present study, we tested this observation in other cancer histologies: breast, colon, lung and pancreatic cancers. Both mRNA and protein expression of FILIP1L were down-regulated in these cancer cells compared with their normal epithelial cells. As in ovarian cancer, DNA methylation is a mechanism by which FILIP1L is down-regulated in these cancer histologies. Methylation status of the FILIP1L promoter was inversely correlated with FILIP1L expression. Reduced methylation in the FILIP1L promoter following treatment with a DNA demethylating agent was associated with restoration of FILIP1L expression in these cancer cells. Further, FILIP1L expression was inversely correlated with the invasive potential of these cancer cells. Re-expression of FILIP1L in FILIP1L-low expressing, highly-invasive cancer cell lines resulted in inhibition of cell invasion. Correspondingly, knockdown of FILIP1L in FILIP1L-high expressing, low-invasive cancer cell lines resulted in increase of cell invasion. Overall, these findings suggest that downregulation of FILIP1L associated with DNA methylation is related with the invasive phenotype in various cancers. Thus, modulation of FILIP1L expression has the potential to be a target for cancer therapy.
\end{abstract}

Citation: Kwon M, Lee SJ, Reddy S, Rybak Y, Adem A, et al. (2013) Down-Regulation of Filamin Ainteracting protein 1 -like Is Associated with Promoter Methylation and an Invasive Phenotype in Breast, Colon, Lung and Pancreatic Cancers. PLoS ONE 8(12): e82620. doi:10.1371/journal.pone.0082620

Editor: Arun Rishi, Wayne State University, United States of America

Received August 1, 2013; Accepted October 25, 2013; Published December 5, 2013

Copyright: @ 2013 Kwon et al. This is an open-access article distributed under the terms of the Creative Commons Attribution License, which permits unrestricted use, distribution, and reproduction in any medium, provided the original author and source are credited.

Funding: This research was supported in part by a generous gift from Linda and Earle Altman and the Albert Einstein College of Medicine. The funders had no role in study design, data collection and analysis, decision to publish, or preparation of the manuscript.

Competing interests: The authors have declared that no competing interests exist.

*E-mail: slibutti@montefiore.org

\section{Introduction}

Cancer metastasis is the most common cause of cancerrelated death, and invasive potential is correlated with poor outcomes in patients with a variety of cancers [1]. Characterization of the cellular mechanisms involved in cancer cell invasion and metastasis will allow for the development of more effective cancer therapies. We identified Filamin A interacting protein 1-like (FILIP1L; previously known as downregulated in ovarian cancer 1 [DOC1]) as an important inhibitor of cell migration and invasion. Increased expression of FILIP1L resulted in inhibition of migration in endothelial cells [2] and inhibition of migration and invasion in cancer cells [3]. FILIP1L expression was inversely correlated with the invasive potential of ovarian cancer cell lines and ovarian cancer specimens [3]. Others have shown that intraperitoneal delivery of the FILIP1L gene resulted in inhibition of metastatic ovarian cancer spread into the peritoneum and intra-abdominal organs [4]. Overall, these findings suggest that FILIP1L may be an important inhibitor of cancer cell invasion and metastasis.

To date, FILIP1L has been shown to be down-regulated only in ovarian and prostate cancers among human cancer histologies. FILIP1L mRNA was originally characterized by its presence in human ovarian surface epithelial (HOSE) cells and its absence in ovarian carcinoma cells [5]. FILIP1L downregulation was confirmed by cDNA microarray analysis in ovarian carcinoma cells from patients with late-stage disease [6]. Differential gene expression analysis revealed that the FILIP1L gene in ovarian cancer cells presents several tagging single nucleotide polymorphisms [7]. FILIP1L was shown to be one of nine genes associated with functional suppression of tumorigenicity in ovarian cancer cell lines [8]. Using cDNA 
microarray analysis, FILIP1L was identified as one of the genes whose transcription is induced in senescent human prostate epithelial cells, but significantly repressed in immortalized prostate epithelial cells $[9,10]$. Recently, we and others have demonstrated that DNA methylation in the FILIP1L promoter was the mechanism by which FILIP1L was down-regulated in ovarian and prostate cancers $[3,11]$.

Based on these observations, we asked whether FILIP1L expression was also down-regulated in other human cancer histologies and whether it was inversely correlated with the degree of invasive potential. In addition, since FILIP1L promoter methylation was associated with FILIP1L downregulation in ovarian and prostate cancers $[3,11]$, we examined whether or not the same mechanism is responsible for the down-regulation of FILIP1L in other cancer histologies.

Our results demonstrate that cellular invasion is inversely correlated with FILIP1L expression in human breast, colon, lung and pancreatic cancer cells. We observed that overexpression of FILIP1L inhibited the invasive potential of aggressive cancer cell lines of these histologies. We also demonstrate that FILIP1L promoter methylation is associated with FILIP1L down-regulation in these cancer cells. Taken together, these data suggest that the degree of FILIP1L expression may be a predictor of cancer cell behavior and, further, that the modulation of FILIP1L expression in various cancers may be a useful target for the development of novel cancer therapies.

\section{Materials and Methods}

\section{Cell culture}

All cell lines used were cultured in RPMI 1640 containing $10 \%$ fetal bovine serum (FBS), unless described separately. Human colon cancer cell lines HT-29, HCT 116, HCT-15, SW620 and T84 were purchased from the American Type Culture Collection (ATCC; Manassas, VA). The other human colon cancer cell lines Caco-2 and SW480, which were originally purchased from ATCC, were provided by Dr. Leonard Augenlicht, Albert Einstein College of Medicine, NY. Human lung cancer cell line $\mathrm{H} 23$ was purchased from ATCC. All the other human lung cancer cell lines - H322 and H1299, H460, A549 and H661, which were originally purchased from SigmaAldrich and ATCC, respectively - were provided by Dr. Roman Perez-Soler, Albert Einstein College of Medicine, NY. Human pancreatic cancer cell lines MIA PaCa-2, PANC-1, Hs 766T, HPAC, HPAF-II, SU.86.86, Panc 02.03 and Capan-1 and human breast cancer cell lines BT-549, Hs 578T, MDAMB-468, BT-474 and ZR-75-1 were purchased from ATCC. The other human breast cancer cell lines MDA-MB-231 and MCF7, which were originally purchased from ATCC, were provided by Dr. Paraic Kenny, Albert Einstein College of Medicine, NY. Immortalized normal human colon cell line NCM460 was obtained from INCELL Corporation (San Antonio) and was cultured in M300F (INCELL) containing 10\% FBS. Human primary cells human small airway epithelial cells (SAEC) and human mammary epithelial cells (HMEC) were purchased from Lonza and were cultured in SAGM and MEGM (Lonza), respectively.

\section{Quantitative real-time RT-PCR}

Various cells, either untreated or DAC- and TSA-treated, were cultured and harvested at $\sim 80 \%$ confluence. Total RNA was prepared by RNeasy kit (Qiagen), and cDNA was prepared by SuperScript VILO cDNA Synthesis Kit (Invitrogen). qRT-PCR was performed using a ViiA7 real-time PCR instrument as recommended by the manufacturer (Applied Biosystems). Expression of the FILIP1L gene was normalized to $h R P L 7$ gene expression. The TaqMan primers used were described previously [3].

\section{Western blot}

Whole cell lysates were prepared from radioimmunoprecipitation assay (RIPA) buffer, separated on SDS-PAGE and transferred to nitrocellulose membrane. The membranes were blotted with antibodies against FILIP1L [2] and glyceraldehyde-3-phosphate dehydrogenase (GAPDH; Chemicon) followed by incubation with anti-mouse antibody conjugated to horseradish peroxidase. The signal was detected using chemiluminescence (Millipore).

\section{Methylation analysis}

Genomic DNAs from various cells were extracted using QIAamp DNA mini kit (Qiagen). Bisulphite modification was performed using EZ DNA Methylation kit (Zymo Research) following manufacturer's instructions. Bisulphite-modified DNA was subjected to nested PCR using HotStar Taq DNA Polymerase kit (Qiagen) following manufacturer's instructions. The nested PCR primers used were described previously [3]. Quantitative DNA methylation was analyzed by Sequenom $\AA$ EpiTYPER Mass Array [12-15]. The assays were performed using the company's standard protocol through Genomics Shared Facility at Albert Einstein College of Medicine, NY. Matched peak data was exported using EpiTYPER software and analyzed quantitatively.

\section{5-Aza-2'-deoxycytidine and Trichostatin A treatment}

Various cancer cells were seeded in 6-well plates at a density of $1-2 \times 10^{5}$ cells per well $16 \mathrm{~h}$ before treatment. Cells were treated with 5-aza-2'-deoxycytidine (DAC; Sigma-Aldrich) daily for $72 \mathrm{~h}$ or with Trichostatin A (TSA; Sigma-Aldrich) once for $24 \mathrm{~h}$.

\section{Cell invasion assay}

Various cells untransfected or transfected with wild-type FILIP1L or FILIP1LAC103 CDNA or FILIP1L SiRNA were cultured at $\sim 80 \%$ confluence. Cells were starved in basal medium containing $0.2 \%$ bovine serum albumin for $16 \mathrm{~h}$. Matrigel invasion was measured using the BD BioCoat Tumor Invasion System (BD Biosciences \#354165) as recommended by the manufacturer. Seeding $4.5 \times 10^{4}$ of starved cells into the apical chambers was followed by adding a chemoattractant (10\% FBS) to the basal chambers. After a $20 \mathrm{~h}$ incubation, quantification of cell invasion was achieved by post-cell invasion labeling with a fluorescent dye, calcein AM (BD Biosciences), and measuring the fluorescence of invading cells of the underside of the membrane at $494 / 517 \mathrm{~nm}$ (excitation/ 
emission). Synergy Mx microplate reader (BioTek) was used to measure fluorescence, and Gen5 software (BioTek) was used to analyzed the data.

\section{Transfection of Cells with FILIP1L plasmids}

Cloning of FILIP1LAC103 (amino acid 1-790) was described previously [2]. Plasmids were purified using Endo-free maxiprep kit (Qiagen). Various cancer cells were transfected with equimolar amounts of control empty plasmid or plasmid encoding wild-type FILIP1L or FILIP1LDC103 using X-fect solution following the manufacturer's protocols (Clontech). After a $24 \mathrm{~h}$ transfection, the cells were subjected to a cell invasion assay.

\section{Transfection of Cells with FILIP1L siRNA}

ON-TARGETplus Non-Targeting SiRNA Pool and SMARTpool of ON-TARGETplus FILIP1L SIRNA was purchased from Thermo Scientific. Various cancer cells were transfected with equimolar amounts of either non-Targeting or FILIP1L siRNA using Dharmafect solution following the manufacturer's protocols (Thermo Scientific). After a $48 \mathrm{~h}$ transfection, the cells were subjected to a cell invasion assay.

\section{Statistical analysis}

Statistical analyses were performed using a two-tailed Student's $t$ test (GraphPad Prism 3.0), and differences were considered statistically significant at a value of $P<0.05$. The correlation of FILIP1L mRNA expression with DNA methylation status of the CpG island in the FILIP1L promoter as well as with invasiveness of the cells was estimated by Spearman's rank correlation method (GraphPad Prism 3.0).

\section{Results}

\section{Differential expression of FILIP1L in various human cancer cell lines}

In order to test the expression levels of FILIP1L in various cancer cells, we measured FILIP1L mRNA expression in several human breast, colon, lung and pancreatic cancer cell lines by qRT-PCR. Normal human primary cells such as human small airway epithelial cells (SAEC) and human mammary epithelial cells (HMEC) as well as immortalized normal human colon cell line (NCM460) were also used. As shown in Figure 1A-C, mRNA expression of FILIP1L was highest in normal primary cells and cell line compared to cancer cell lines. For pancreatic cancer, normal cells were not included since they were not available. Interestingly, breast cancer cell line MCF7, which has been shown to be a non-invasive cell line [16], demonstrated as high FILIP1L expression as normal breast epithelial cells HMEC. In order to test if the protein expression of FILIP1L is correlated with its mRNA expression in these various cancer cells, we measured FILIP1L protein expression by immunoblot analysis using anti-FILIP1L antibody. As shown in Figure 2, FILIP1L protein expression was much higher in cells that express higher FILIP1L mRNA. FILIP1L protein was undetectable in many cancer cell lines. Interestingly, normal epithelial cells HMEC and SAEC did not show a robust FILIP1L protein band despite higher FILIP1L mRNA expression. These data demonstrate that the protein expression of FILIP1L is correlated with its mRNA expression in most cells from these histologies. Thus, these findings suggest that FILIP1L expression is down-regulated in various cancer cell lines compared with a normal cell line and non-invasive cancer cell lines.

\section{Inverse correlation of FILIP1L expression with the FILIP1L promoter methylation in various human cancer cell lines}

We previously showed that the DNA methylation status at the FILIP1L promoter inversely correlated with FILIP1L expression [3]. As previously discussed, the average overall methylation was measured for the analyzed 21 CG sites out of total 59 CG sites in the CpG island of the FILIP1L promoter [3]. The average overall methylation demonstrated that these CG sites were highly methylated in cancer cells including BT-549 and Hs 578T breast cancer cells, HT-29, HCT 116 and HCT-15 colon cancer cells, H1299 lung cancer cells and MIA PaCa-2 pancreatic cancer cells (Figure 3A-D). In contrast, these sites were unmethylated in normal cells (HMEC, NCM460 and SAEC) as well as some cancer cell lines including MCF7 breast cancer cells, SW620 colon cancer cells and SU.86.86, Panc 02.03 and Capan-1 pancreatic cancer cells (Figure 3A$D)$. We tested if the DNA methylation status of the $\mathrm{CpG}$ island of the FILIP1L promoter (Figure 3A-D) inversely correlated with FILIP1L mRNA expression (Figure 1). As shown in Figure 3E$\mathrm{H}$, a significant inverse correlation was present in all four cancer histologies, suggesting that DNA methylation in the FILIP1L promoter may mediate FILIP1L down-regulation in various cancer histologies.

\section{Association of reduced methylation in the FILIP1L promoter with restoration of FILIP1L expression in various cancer cells following treatment with a DNA demethylating agent}

To further test if epigenetic regulation results in reexpression of FILIP1L, we treated the four lowestFILIP1Lexpressing cancer cell lines from each cancer histology (BT-549, HT-29, H1299 and MIA PaCa-2; Figure 1) with either a DNA demethylating agent or histone deacetylase inhibitor. Treatment of these cells with DNA demethylating agent 5aza-2'-deoxycytidine (DAC) resulted in significantly increased FILIP1L mRNA expression in all four cancer histologies (Figure 4). Interestingly, histone deacetylase inhibitor Trichostatin $A$ (TSA) treatment also resulted in significantly increased FILIP1L mRNA expression in these cancer cell lines, except for the BT-549 breast cancer cell line. FILIP1L mRNA expression in DAC- or TSA-treated cells was compared with that in DMSO control-treated cells. In order to examine whether reduced methylation in the FILIP1L promoter is associated with restoration of FILIP1L expression, we analyzed the methylation status of the FILIP1L promoter in the cells utilized in Figure 4 following DAC treatment. Most CG sites analyzed in the $\mathrm{CpG}$ island of the FILIP1L promoter demonstrated similar reduction in percent methylation in each cell type (data not shown). As shown in Figure 5, all four cell lines treated with DAC 
A

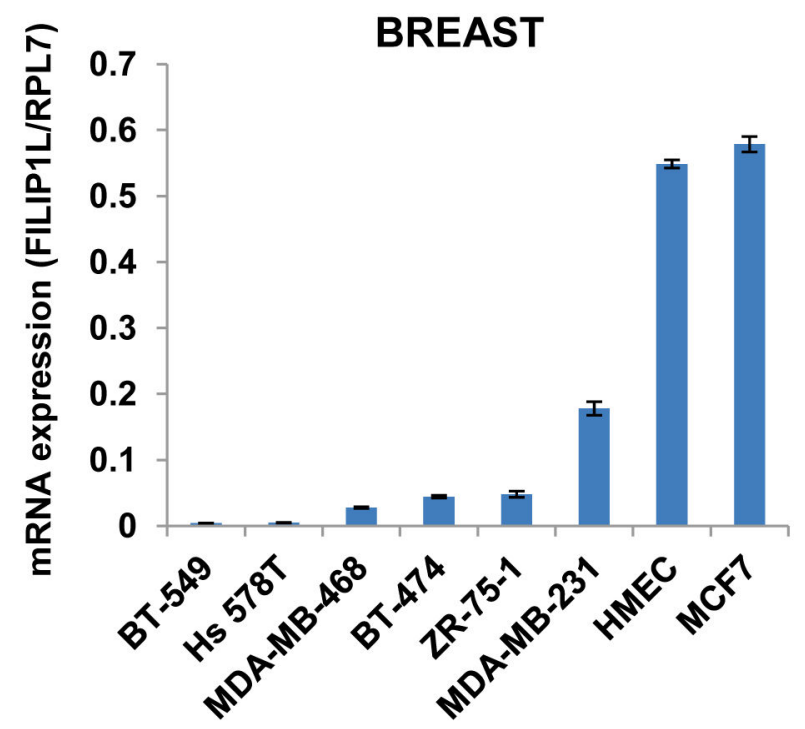

C

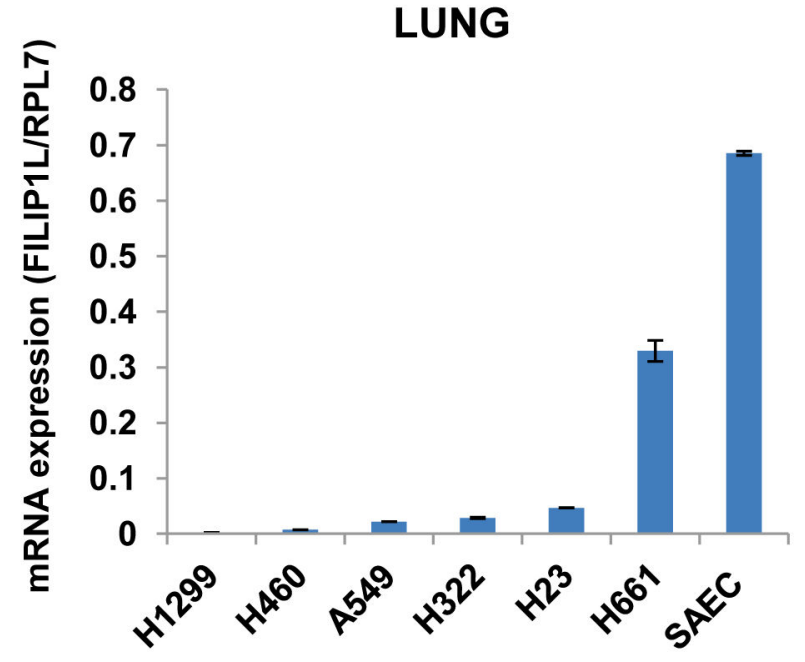

B
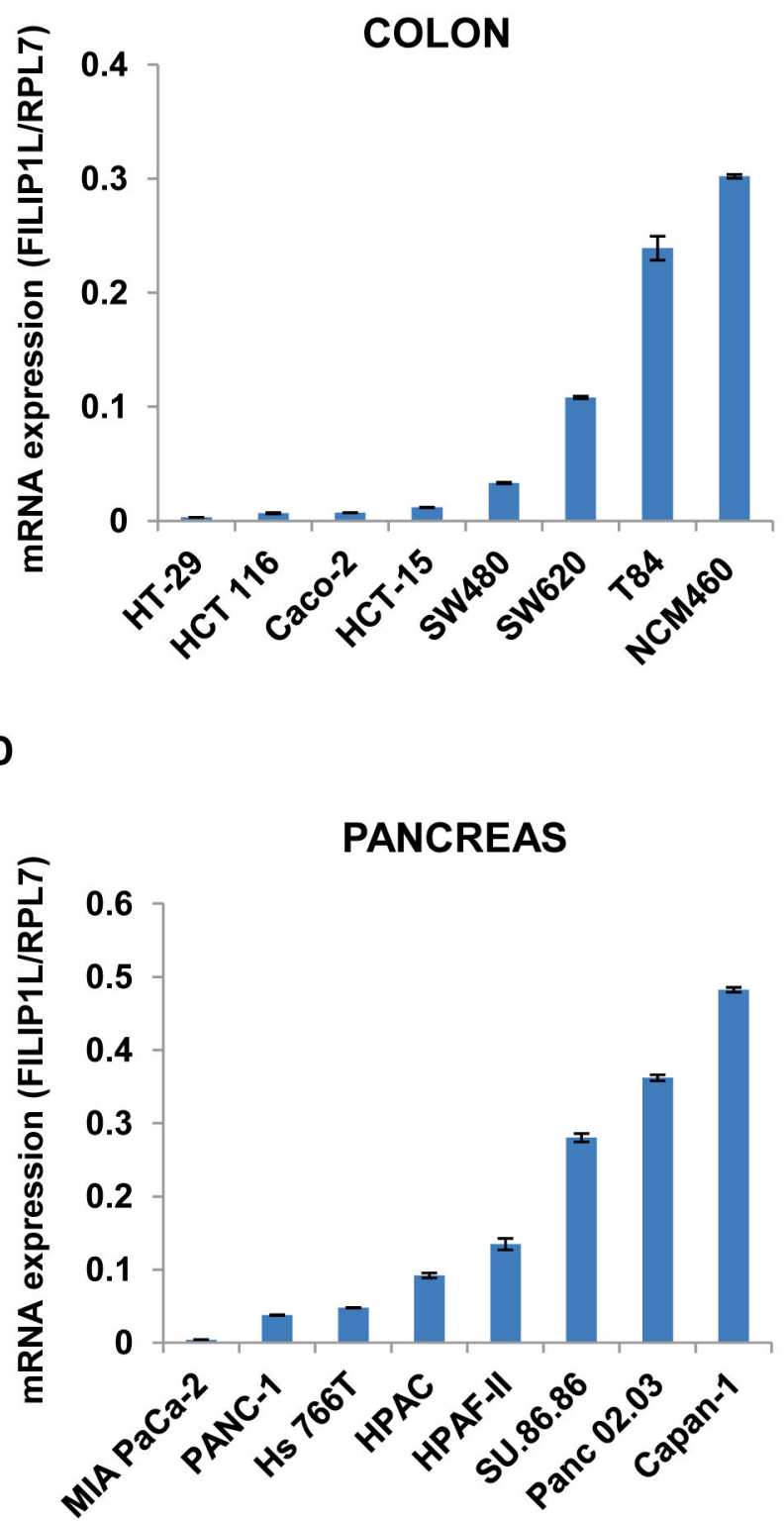

Figure 1. Differential expression of FILIP1L mRNA in various human cancer cell lines. qRT-PCR analysis for FILIP1L conducted on cDNA from human breast $(A)$, colon $(B)$, lung $(C)$ and pancreatic $(D)$ cancer cell lines. The $y$ axis represents FILIP1L mRNA expression which was standardized with the housekeeping gene $h R P L 7$. Error bars indicate SEM $(n=3)$. The result is an average of three independent experiments.

doi: 10.1371/journal.pone.0082620.g001

demonstrated a significant decrease in the average overall methylation. These data demonstrate that DNA methylation in the FILIP1L promoter is a mechanism by which FILIP1L is down-regulated in various cancer histologies.
Inverse correlation of FILIP1L expression with the invasive potential of various human cancer cell lines

We previously demonstrated that the down-regulation of FILIP1L expression associated with DNA methylation in the FILIP1L promoter is related with invasive potential in ovarian cancer cell lines [3]. We tested whether FILIP1L expression is inversely correlated with the invasive potential of breast, colon, lung and pancreatic cancer cell lines. We examined the invasive activity for the same cell lines utilized in Figure 1 by 
A

BREAST
B
COLON
C

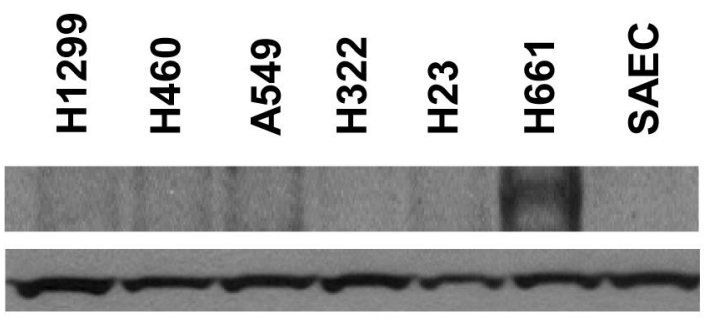

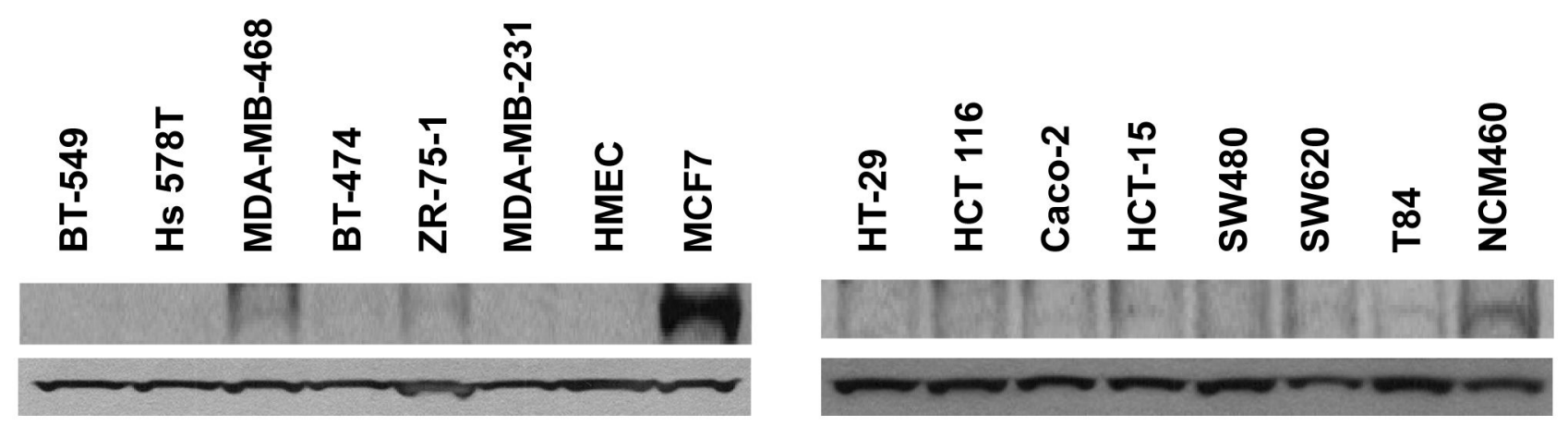

D PANCREAS

Figure 2. Differential expression of FILIP1L protein in various human cancer cell lines. Immunoblot analysis for FILIP1L in the same cells utilized in Figure 1. GAPDH blot is shown as the loading control. The result is representative of three independent experiments.

doi: 10.1371/journal.pone.0082620.g002

Matrigel invasion assay. As shown in Figure 6A-D, most of the cell lines that demonstrated low FILIP1L expression invaded Matrigel significantly more than those that demonstrated high FILIP1L expression. In addition, the FILIP1L mRNA expression demonstrated a significant inverse correlation with invasiveness of the cells (Figure 6E-H), suggesting that FILIP1L expression is inversely correlated with the invasive potential of all four cancer cell lines. We then tested if overexpression of FILIP1L in FILIP1L-low-expressing, highlyinvasive cancer cell lines resulted in inhibition of cell invasion. The same cell lines utilized in Figure 4 and 5 were tested. Our previous studies have shown that overexpression of FILIP1L $\triangle \mathrm{C} 103(\mathrm{COOH}$ terminal truncation mutant 1-790) as well as wild-type FILIP1L resulted in inhibition of ovarian cancer cell invasion [3]. Thus, we transfected these various cancer cells with a plasmid encoding control, wild-type FILIP1L or FILIP1LAC103 CDNA, and measured invasion. Under the same experimental condition, the expression level of FILIP1L $\triangle$ C103 was higher than that of wild-type FILIP1L in all four cell lines (Figure 7A). As shown in Figure 7B-E, all four cell lines transfected with either wild-type FILIP1L or FILIP1LAC103 cDNA invaded Matrigel significantly less than those transfected with control. To definitively prove that FILIP1L inversely regulates invasive properties of the cancer cells, we tested if knockdown of FILIP1L in FILIP1L-high expressing, low-invasive cancer cell lines resulted in an increase in cell invasion. We transfected these various cancer cells with either non-targeting or FILIP1L siRNA and measured invasion. As shown in Figure 8A, all four cell lines transfected with FILIP1L siRNA demonstrated a reduction of FILIP1L compared with control. All four cell lines transfected with FILIP1L siRNA invaded Matrigel significantly more than those transfected with control siRNA (Figure 8B-E). Collectively, these data suggest that down-regulation of FILIP1L is associated with an invasive phenotype in various cancer cell lines and that this phenotype can be reversed by overexpression of FILIP1L. 
A

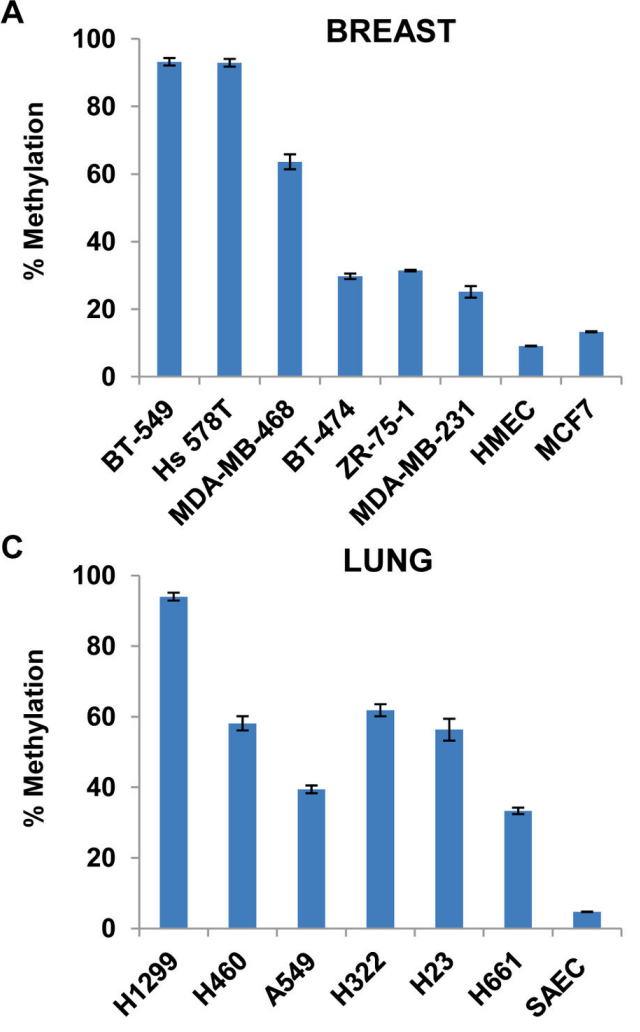

E

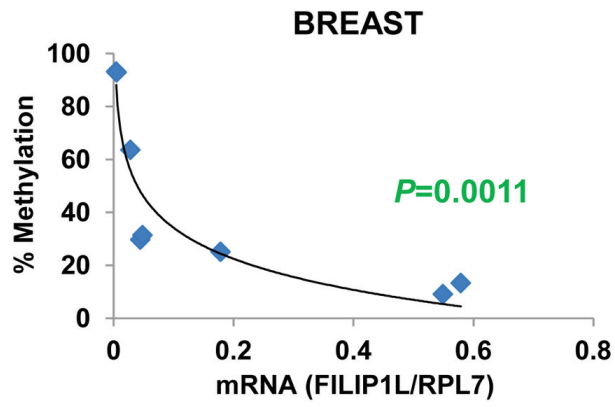

G

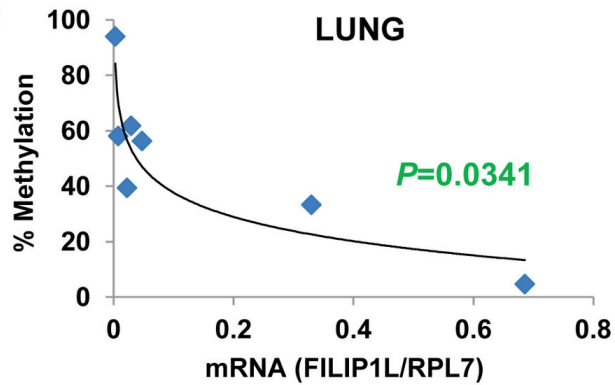

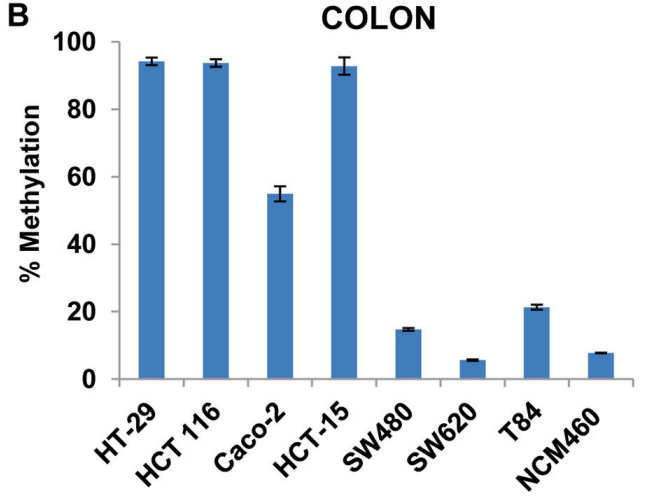

D
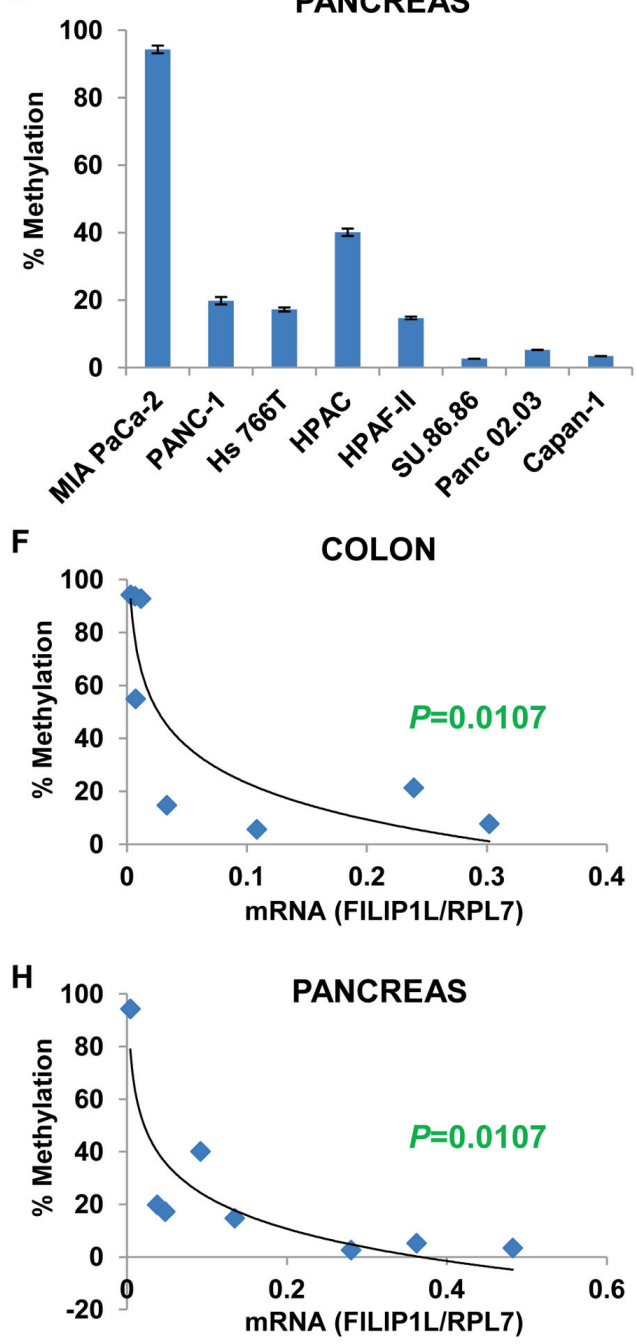

Figure 3. Inverse correlation of FILIP1L expression with FILIP1L promoter methylation in various human cancer cell lines. $A-D$, DNA methylation status of the CpG island in the FILIP1L promoter from the same cell lines utilized in Figure 1 was analyzed by Sequenom $®$ EpiTYPER Mass Array. Mass Array results are shown as the average overall methylation for the analyzed 21 CG sites out of total 59 CG sites in the CpG island of the FILIP1L promoter. Error bars indicate SEM $(n=3)$. E-H, A significant inverse correlation of the DNA methylation status of the CpG island in the FILIP1L promoter with FILIP1L mRNA expression (each $P$ value was calculated by Spearman's rank correlation method). y axis: percent methylation of the average overall methylation for all 21 CG sites shown in sections $A-D$ was used. $\mathrm{x}$ axis: standardized FILIP1L mRNA expression shown in Figure 1 was used.

doi: 10.1371/journal.pone.0082620.g003 
A

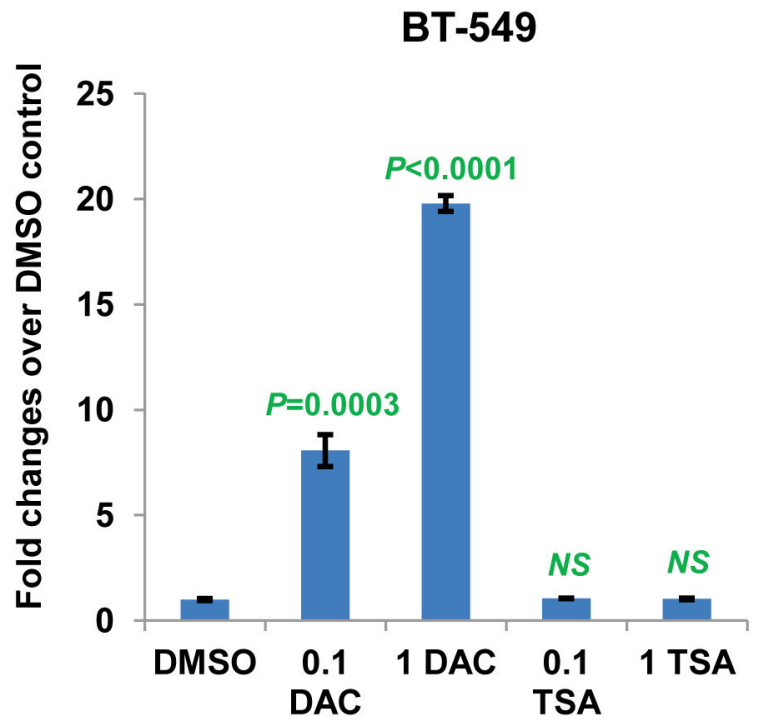

C

\section{H1299}

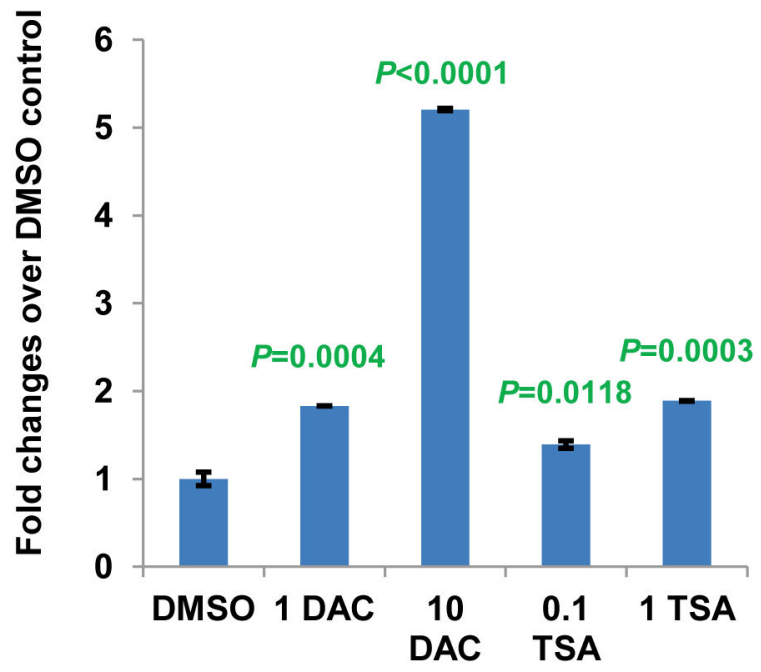

B

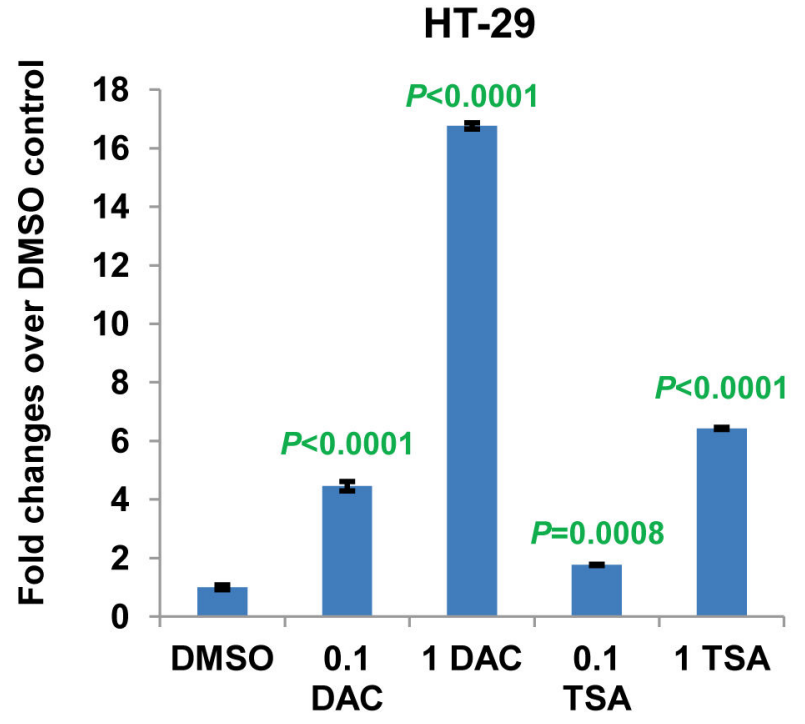

D

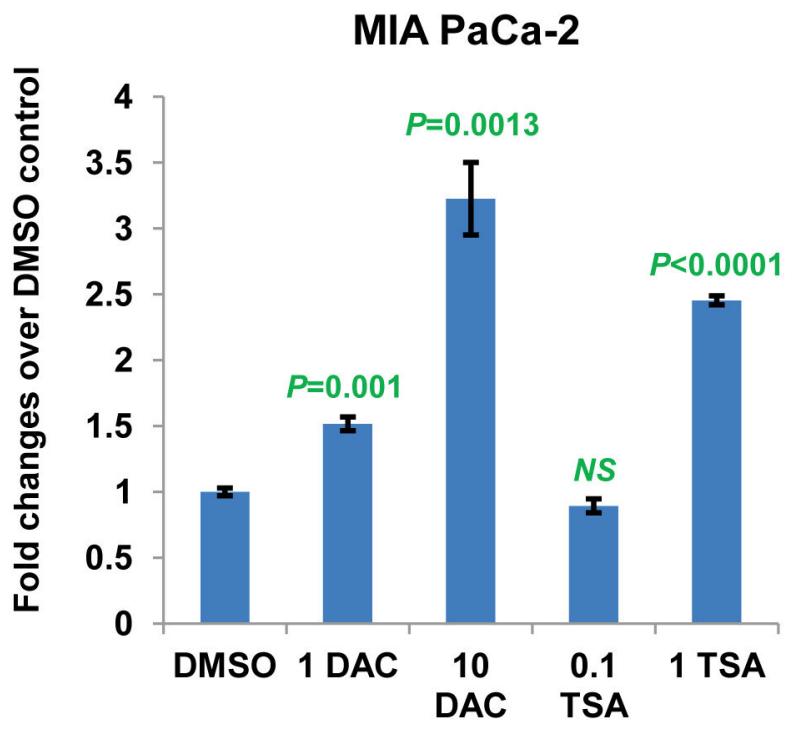

Figure 4. Restoration of FILIP1L expression in various cancer cells following treatment with a DNA demethylating agent or a histone deacetylase inhibitor. qRT-PCR analysis for FILIP1L conducted on CDNA from BT-549 breast $(A)$, HT-29 colon (B), H1299 lung $(C)$ and MIA PaCa-2 pancreatic $(D)$ cancer cell lines treated with either 5-aza-2'-deoxycytidine (DAC) or Trichostatin A (TSA). Values $0.1,1$ or 10 in the $x$ axis indicate the concentration of DAC and TSA in $\mu M$. The $y$ axis represents fold change of each reagent-treated cell type over DMSO-treated control cells, where each value was standardized with the housekeeping gene $h R P L 7$. Error bars indicate SEM $(n=3)$. The result is an average of two independent experiments. $P$ values are derived from comparison between DMSO-treated control and either DAC- or TSA-treated experiments. NS indicates not significant.

doi: 10.1371/journal.pone.0082620.g004

\section{Discussion}

Cancer cell invasion is a critical first step in cancer metastasis, and the invasive potential of cancer cells is correlated with poor outcomes in patients with a variety of cancers [1]. Characterization of the cellular mechanisms involved in invasion will allow for the development of more effective cancer therapies. We previously demonstrated that FILIP1L expression was inversely correlated with the invasive potential of ovarian cancer cell lines and ovarian cancer specimens [3]. In the present study, we have shown that FILIP1L expression is inversely correlated with the invasive 
A

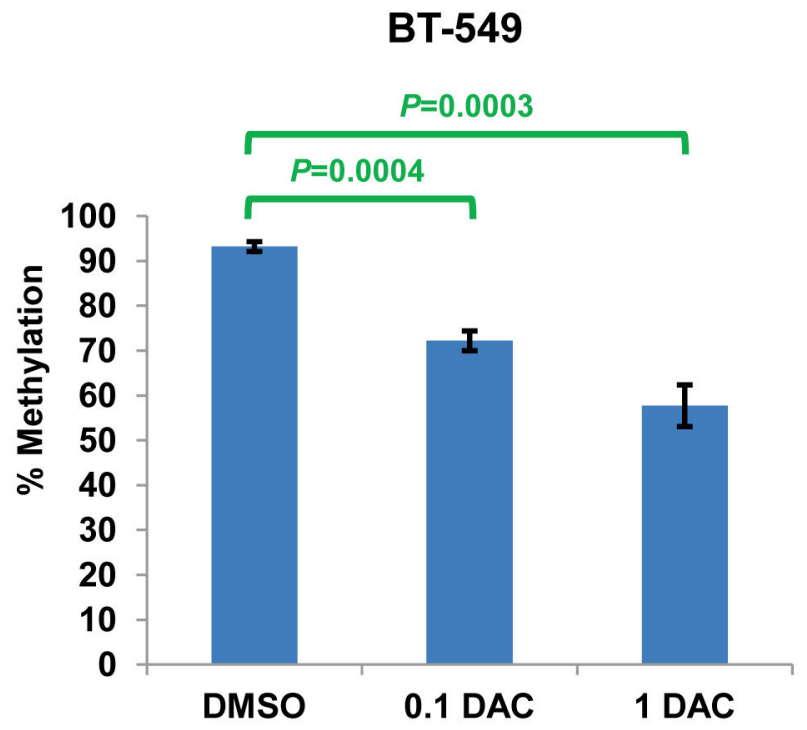

C

\section{H1299}

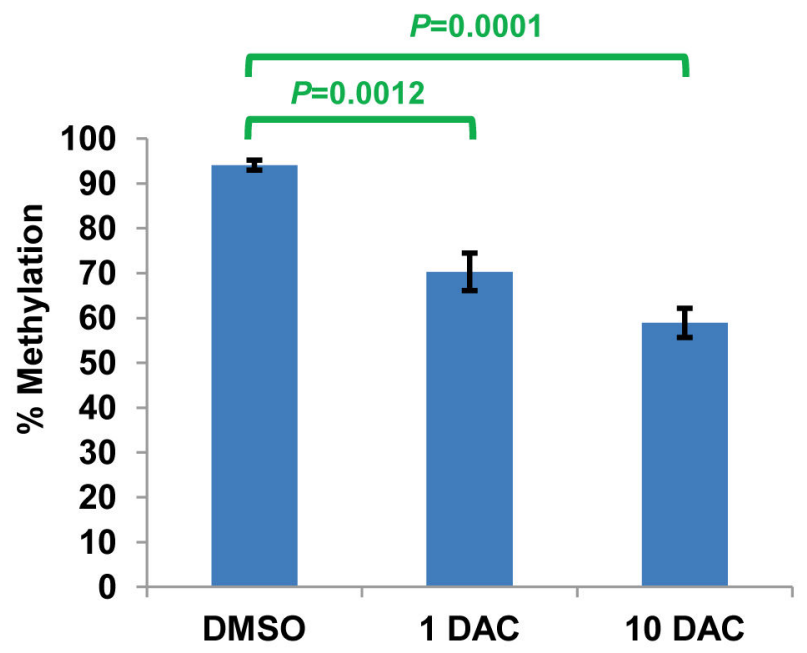

B

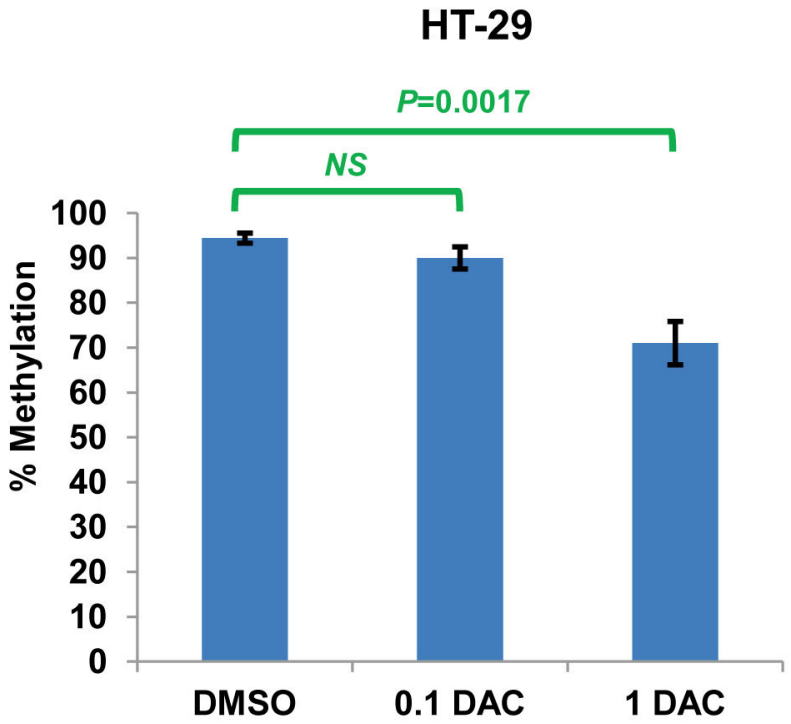

D

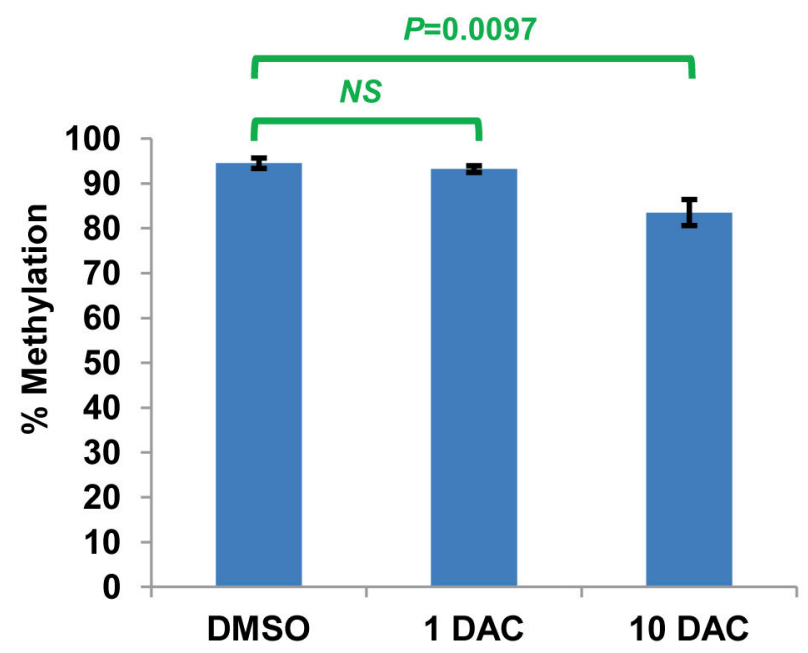

Figure 5. Association of reduced methylation in the FILIP1L promoter with restoration of FILIP1L expression in various cancer cells following treatment with a DNA demethylating agent. DNA methylation status of the CpG island in the FILIP1L promoter from the same cells used in Figure 4 was analyzed by Sequenom® EpiTYPER Mass Array. Mass Array results are shown as described in Figure 3A-D. The result is an average of two independent experiments. $P$ values are derived from comparison between DMSO-treated control and each DAC-treated experiment.

doi: 10.1371/journal.pone.0082620.g005

potential of various cancer cell lines such as breast, colon, lung and pancreatic cancer. Others have shown that FILIP1L expression was down-regulated in prostate cancers compared with normal tissues [11]. Thus, findings from the present study suggest that FILIP1L could be down-regulated and inversely correlated with the invasive potential of many human cancer histologies. Furthermore, it was recently shown that intraperitoneal delivery of the FILIP1L gene resulted in inhibition of metastatic ovarian cancer spread into the peritoneum and intra-abdominal organs [4]. Overall, these findings suggest that FILIP1L may be an important inhibitor of cancer cell invasion and metastasis in a wide variety of tumor types. Therefore, it will be of interest to identify the mechanism by which FILIP1L inhibits cancer cell invasion and metastasis in these various cancer histologies. 
A

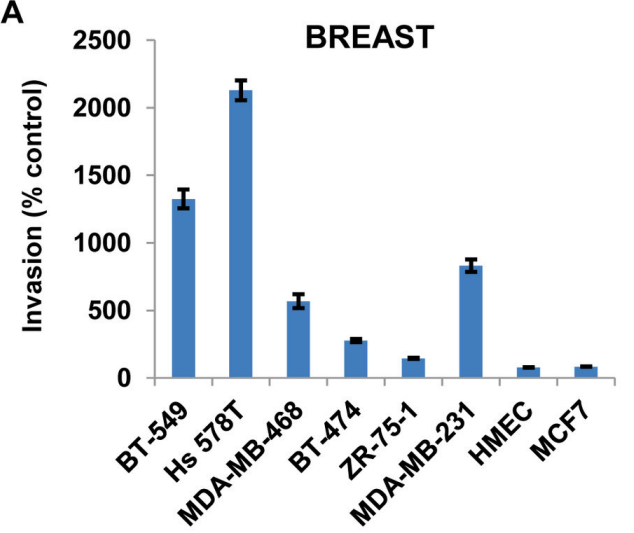

C

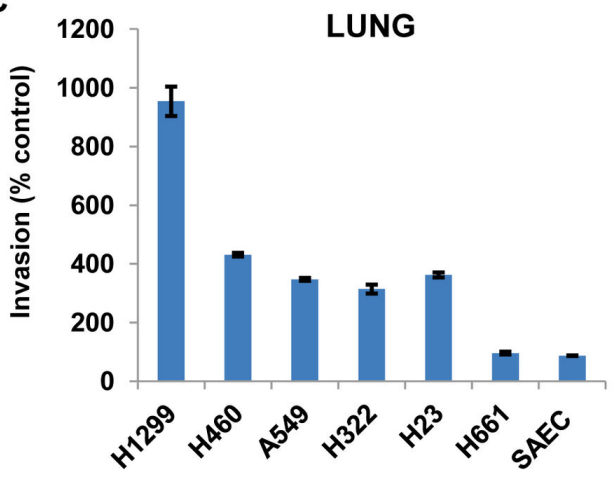

E

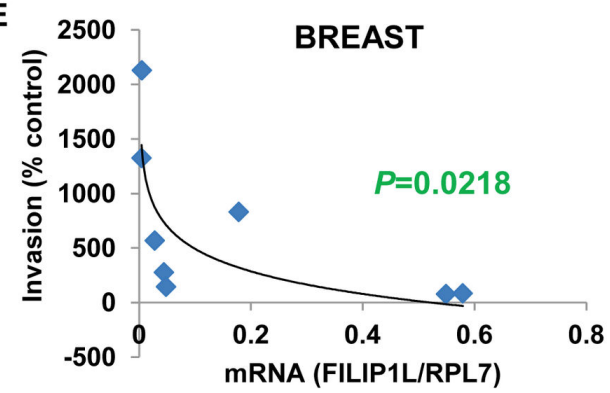

G

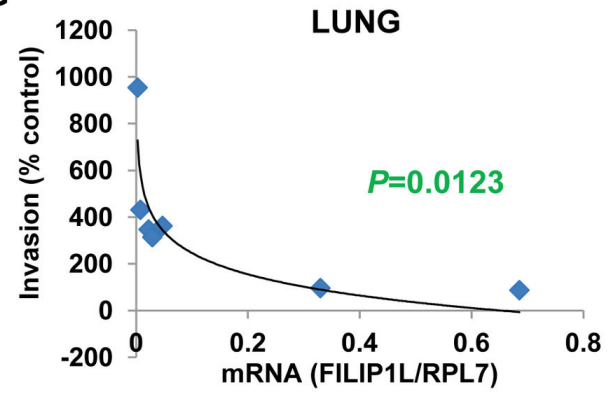

B
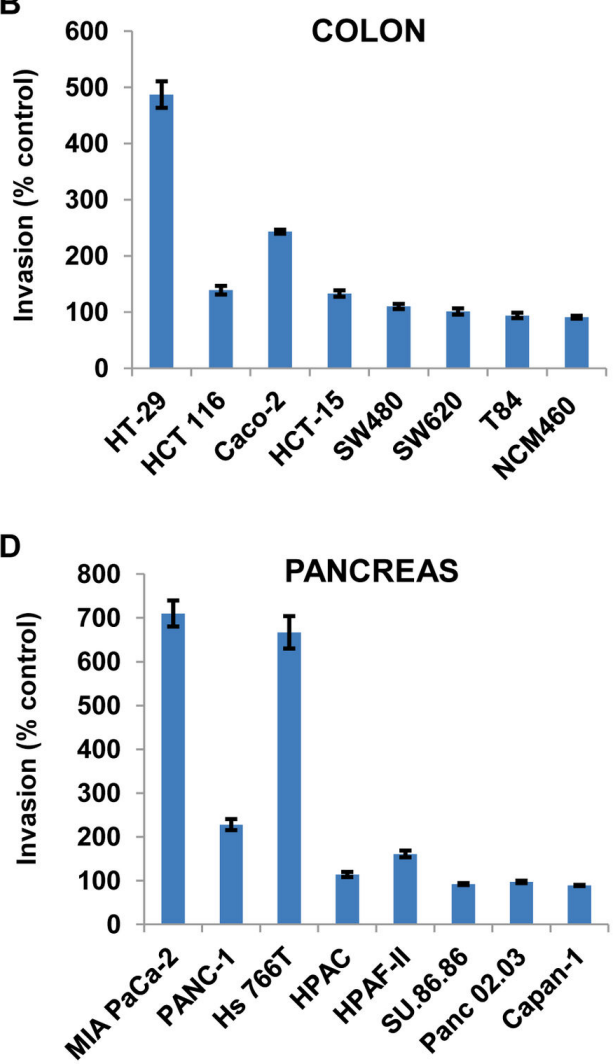

$\mathbf{F}$

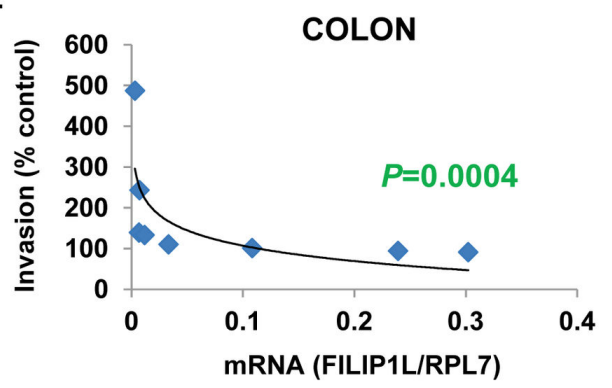

H

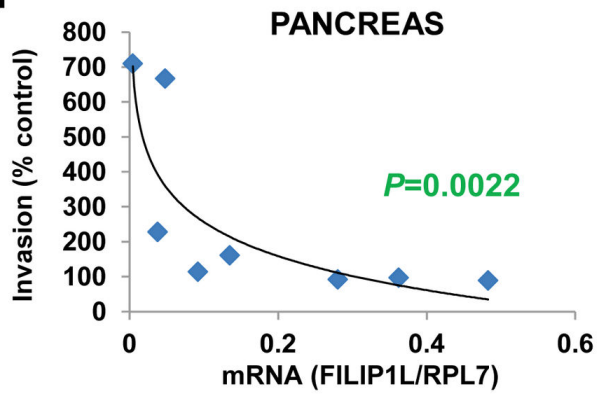

Figure 6. Inverse correlation of FILIP1L expression with the invasive potential of various human cancer cell lines. $A-D$, Matrigel cell invasion assay for the same cells utilized in Figure 1. Matrigel invasion was measured using the BD BioCoat Tumor Invasion System as described in Materials and Methods. The $y$ axis represents a percent change over serum-free control. Error bars indicate SEM $(n=4)$. The result is representative of three independent experiments. $E-H, A$ significant inverse correlation of the FILIP1L mRNA expression with invasiveness of the cells (each $P$ value calculated by Spearman's rank correlation method). $y$ axis: invasiveness of the cells as a percent change over serum-free control shown in sections $A-D$ was used. $\mathrm{x}$ axis: standardized FILIP1L mRNA expression shown in Figure 1 was used.

doi: 10.1371/journal.pone.0082620.g006 
A

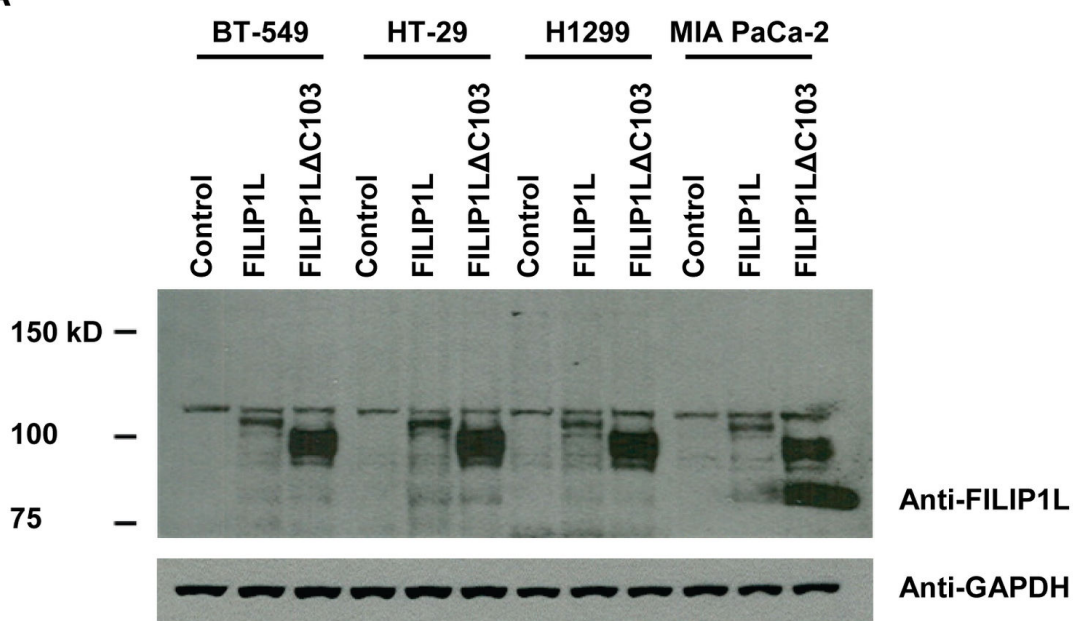

B
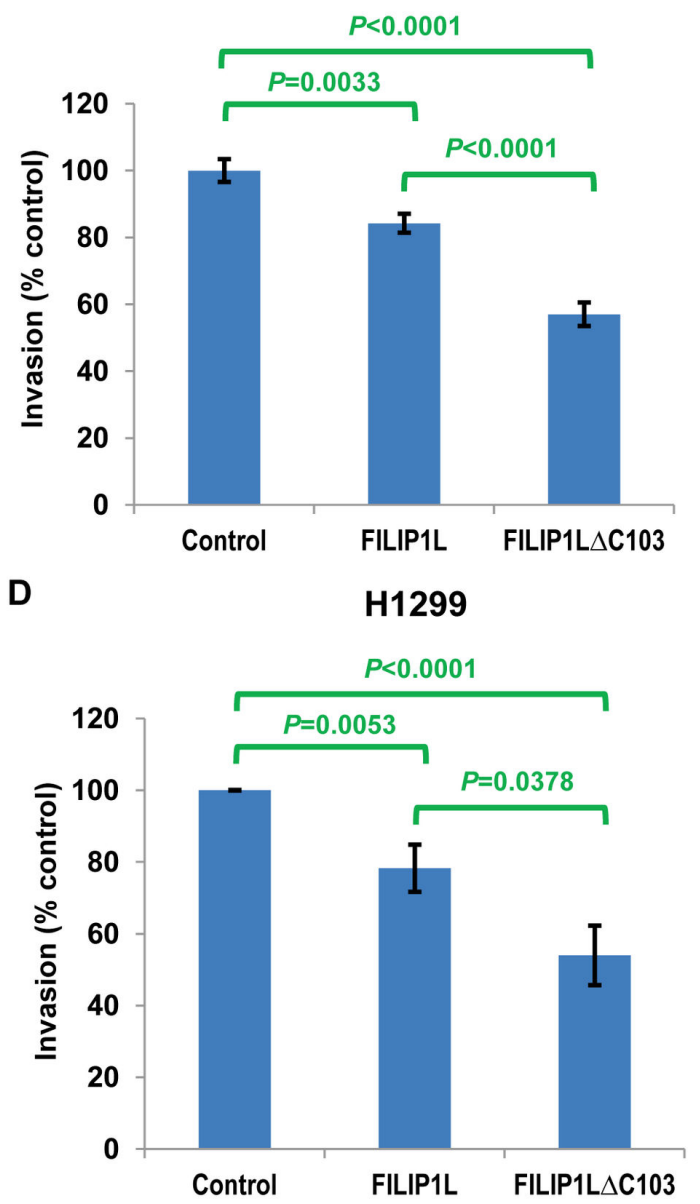

C
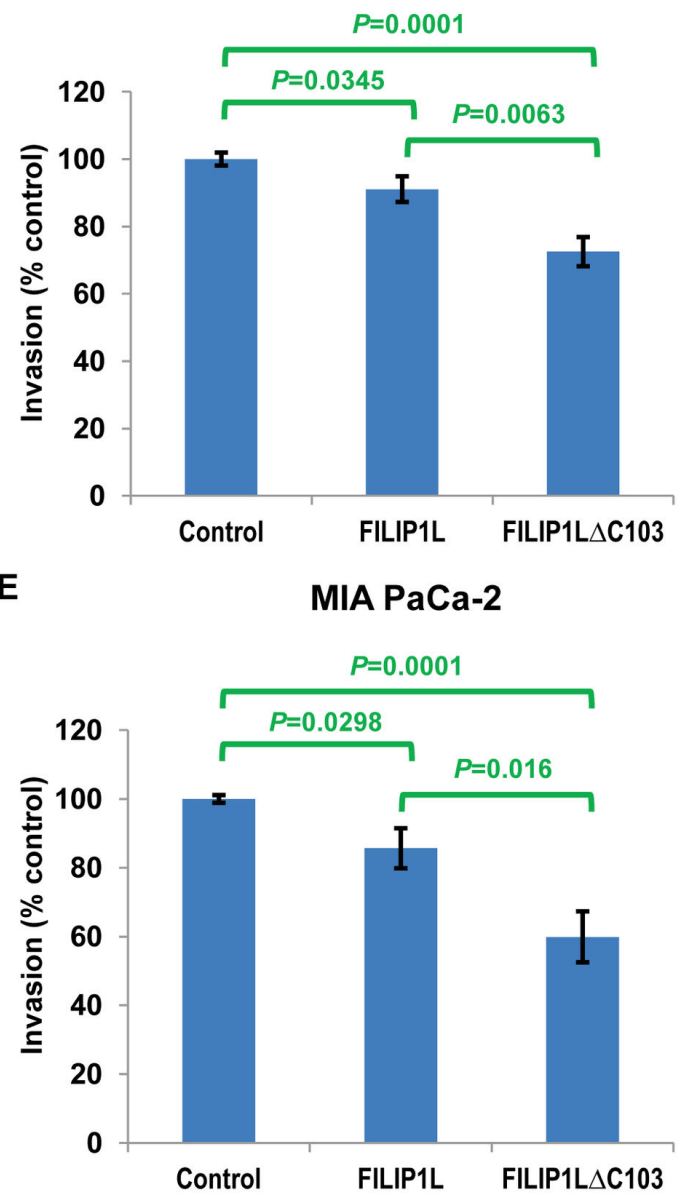

Figure 7. Inhibition of cell invasion following FILIP1L expression in various FILIP1L-low-expressing, highly-invasive cancer cell lines. The same cell lines used in Figure 4 were transfected with control, wild-type FILIP1L or FILIP1LAC103 cDNA. A, Immunoblot analysis for FILIP1L in the transfected cells. GAPDH blot is shown as the loading control. $B-E$, Transfected cells were subject to Matrigel cell invasion assay $24 \mathrm{~h}$ after transfection. The same experimental procedures were followed as described in Figure 6. The result is representative of two independent experiments.

doi: 10.1371/journal.pone.0082620.g007 
A

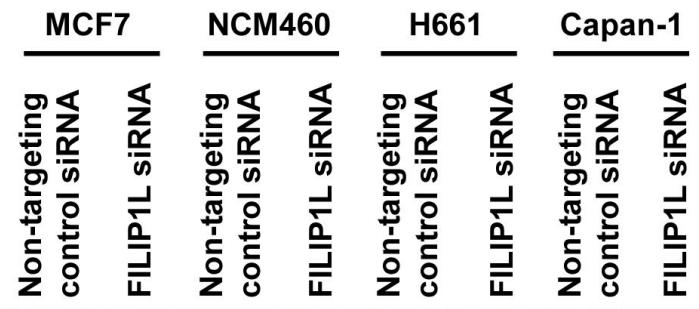

Anti-FILIP1L

Anti-GAPDH

B

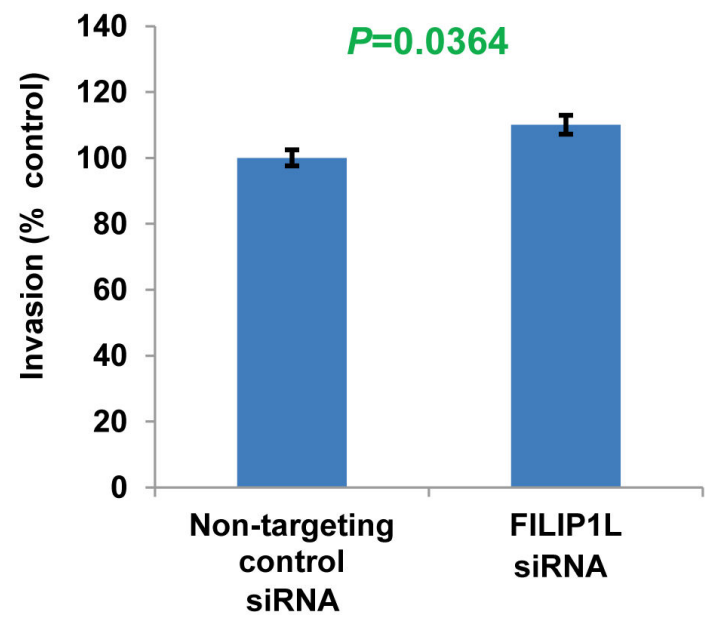

D

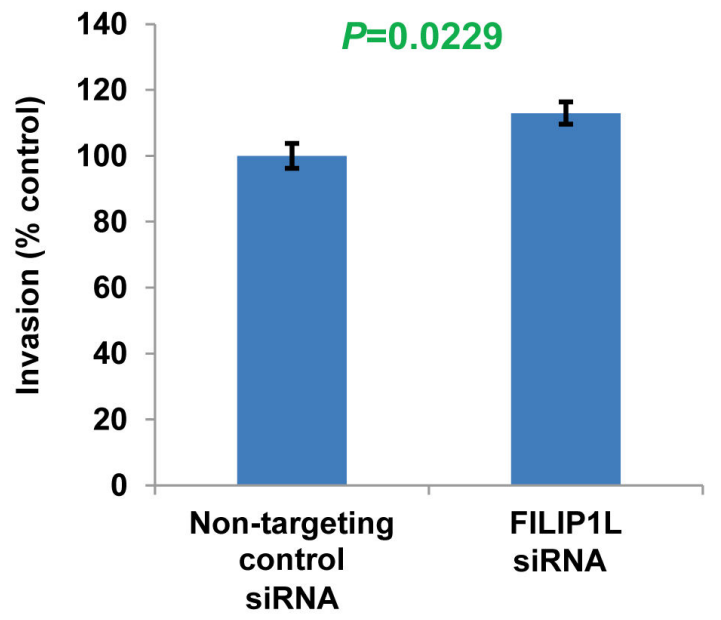

C

NCM460

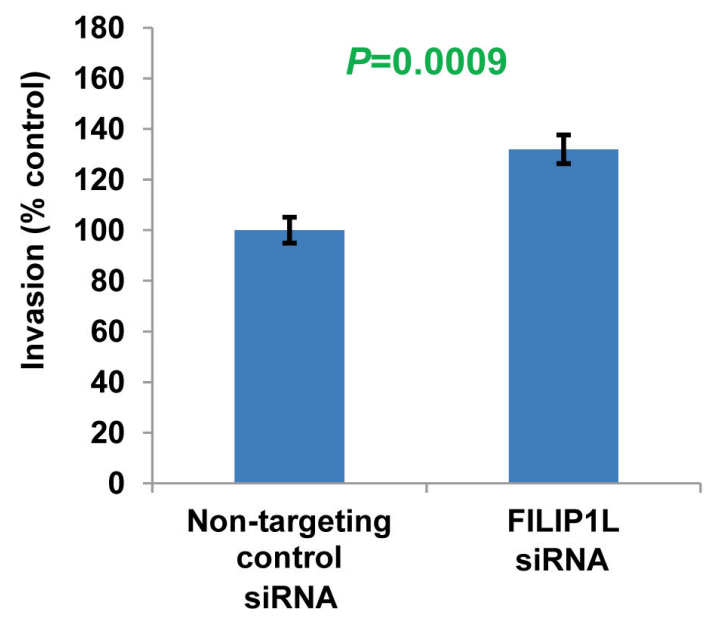

E

\section{Capan-1}

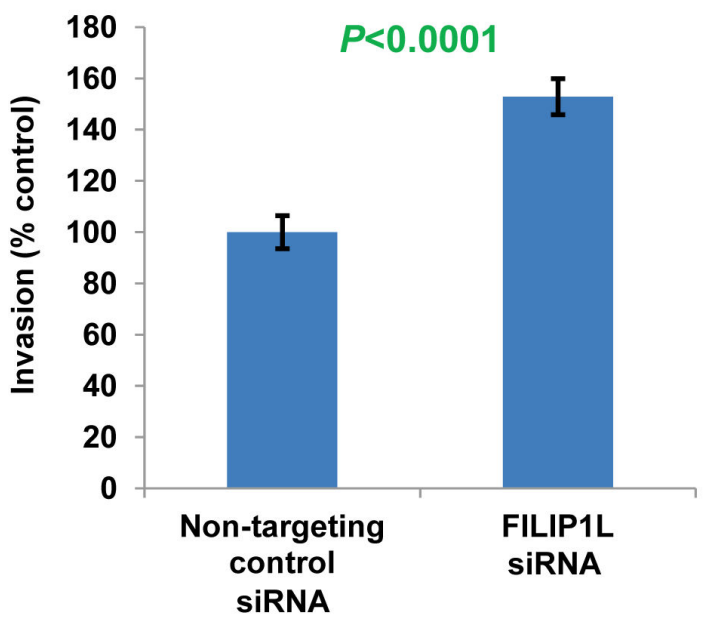

Figure 8. Increase of cell invasion following FILIP1L knockdown in various FILIP1L-high-expressing, low-invasive cancer cell lines. MCF7 breast $(B)$, NCM460 colon $(C)$, H661 lung $(D)$ and Capan-1 pancreatic $(E)$ cancer cell lines were transfected with either non-targeting or FILIP1L siRNA. A, Immunoblot analysis for FILIP1L in the transfected cells. GAPDH blot is shown as the loading control. $B-E$, Transfected cells were subject to Matrigel cell invasion assay $48 \mathrm{~h}$ after transfection. The same experimental procedures were followed as described in Figure 6. The result is representative of two independent experiments.

doi: 10.1371/journal.pone.0082620.g008 
We initially identified FILIP1L in the setting of tumor angiogenesis. In order to identify common intracellular mediators of proliferation, migration and apoptosis in endothelial cells, we previously analyzed gene expression profiles of endothelial cells after treatment with angiogenesis inhibitors such as endostatin, fumagillin and EMAP-II $[17,18]$. FILIP1L was up-regulated in endothelial cells in response to these inhibitors. We subsequently demonstrated that overexpression of FILIP1L resulted in inhibition of cell proliferation and migration and increased apoptosis in endothelial cells [2]. In addition, targeted expression of FILIP1L in the tumor vasculature inhibited tumor growth in vivo [2]. These findings suggested that FILIP1L may be an important inhibitor of cell proliferation and migration as well as an inducer of apoptosis in endothelial cells. Along with the findings from the present study, it suggests that FILIP1L may be utilized as a cancer therapeutic, which can inhibit cancer metastasis as well as angiogenesis.

Normal epithelial cells HMEC and SAEC did not show FILIP1L protein expression despite higher FILIP1L mRNA expression. When we previously performed the same experiments using normal ovarian epithelial cells, the protein level strongly correlated with mRNA level [3]. This discrepancy could be explained by posttranscriptional mRNA degradation, translational efficiency and/or protein degradation, which have been shown to be dynamic and cell specific [19-24]. Currently, we do not have an explanation for the lack of FILIP1L protein detection, and we are exploring this phenomenon further. Understanding a cell-type specific regulation of FILIP1L protein expression may give us insights into the regulation of this antiinvasive protein.

FILIP1L $\triangle$ C103, a $\mathrm{COOH}$ terminal truncation mutant of FILIP1L demonstrated a greater anti-invasion activity than the wild-type FILIP1L. The wild-type FILIP1L has 893 amino acids. As previously described [2], the $\mathrm{NH} 2$ terminal half of the FILIP1L protein consists of a coiled-coil region (residues 3-542), two leucine zipper motifs (residues 83-111 and 218-253) and a prefoldin domain (residues 465-535), and has a SbcC (COG0419; ATPase involved in DNA repair; residues 19-576) conserved domain. Its $\mathrm{COOH}$ terminal half is an unstructured region and has a Herpes_BLLF1 (pfam05109; Herpes virus major outer envelope glycoprotein; residues 640-829) conserved domain. As shown in Figure 7A, the expression level of FILIP1L $\triangle \mathrm{C} 103$ was greater than that of wild-type FILIP1L, which may explain the observation of a greater anti-invasive activity of FILIP1L $\Delta$ C103 compared to wild-type FILIP1L. The same results were observed when ovarian cancer cells were tested previously [3]. Deleting 103 amino acids from the $\mathrm{COOH}$ terminus of FILIP1L greatly enhanced protein stability in cancer cells. Whether disrupting a Herpes_BLLF1 conserved domain is directly involved in enhancing FILIP1L's stability is not currently known.
We previously showed that FILIP1L expression was significantly lower in invasive serous carcinoma than in noninvasive serous borderline tumors [3]. The methylation of the CpG island in the FILIP1L promoter was significantly higher in invasive serous carcinomas than that in non-invasive serous borderline tumors [3]. Others have shown that FILIP1L expression was significantly lower and that FILIP1L promoter methylation was significantly higher in prostate carcinoma samples compared with matched normal tissues [11]. Thus, we believe it would be important to confirm if FILIP1L is downregulated in tissues of other cancer histologies such as breast, colon, lung and pancreas, and whether or not FILIP1L promoter methylation is a mechanism by which FILIP1L is down-regulated in these cancer tissues.

We have shown that a DNA demethylating agent DAC, but not the histone deacetylase inhibitor TSA, resulted in increased FILIP1L mRNA in FILIP1L-low-expressing ovarian [3] and breast (Figure 4A) cancer cells, suggesting that DNA methylation, but not histone modification, is associated with the down-regulation of FILIP1L in these cells. However, TSA treatment also resulted in increased FILIP1L mRNA in cancer cells from other histologies such as colon, lung and pancreas (Figure 4B-D), which suggests that DNA methylation is not the only mechanism by which FILIP1L is down-regulated in these cells. It will be necessary to further examine whether there is an additive effect of DNA methylation and histone modification on FILIP1L down-regulation. In addition, examination of the potential preference of either or both mechanisms by celland/or tissue-specificity will also improve the understanding of how FILIP1L is down-regulated.

\section{Acknowledgements}

In summary, we have demonstrated that down-regulation of FILIP1L is associated with the invasive phenotype in cancer cells of various histologies. FILIP1L promoter methylation is associated with down-regulation of FILIP1L in these cancer cells. Further characterization of the mechanism of FILIP1L down-regulation may provide us with the understanding of the role played by FILIP1L in carcinogenesis and lead to the development of more effective anticancer therapies.We thank Dr. Leonard Augenlicht for providing Caco-2 and SW480, and Dr. Roman Perez-Soler for providing H1299, H460, A549, $\mathrm{H} 322$, and H661 and Dr. Paraic Kenny for providing MDAMB-231 and MCF7.

\section{Author Contributions}

Conceived and designed the experiments: MK SKL. Performed the experiments: MK SJL SR YR AA. Analyzed the data: MK. Wrote the manuscript: MK SKL. 


\section{References}

1. Bravo-Cordero JJ, Hodgson L, Condeelis J (2012) Directed cell invasion and migration during metastasis. Curr Opin Cell Biol 24: 277-283. doi:10.1016/j.ceb.2011.12.004. PubMed: 22209238

2. Kwon M, Hanna E, Lorang D, He M, Quick JS et al. (2008) Functional characterization of filamin a interacting protein 1-like, a novel candidate for antivascular cancer therapy. Cancer Res 68: 7332-7341. doi: 10.1158/0008-5472.CAN-08-1087. PubMed: 18794120.

3. Burton ER, Gaffar A, Lee SJ, Adeshuko F, Whitney KD et al. (2011) Downregulation of Filamin A interacting protein 1-like is associated with promoter methylation and induces an invasive phenotype in ovarian cancer. Mol Cancer Res 9: 1126-1138. doi: 10.1158/1541-7786.MCR-11-0162. PubMed: 21693594.

4. Xie C, Gou ML, Yi T, Deng H, Li ZY et al. (2011) Efficient inhibition of ovarian cancer by truncation mutant of FILIP1L gene delivered by novel biodegradable cationic heparin-polyethyleneimine nanogels. Hum Gene Ther 22: 1413-1422. doi:10.1089/hum.2011.047. PubMed: 21513424

5. Mok SC, Wong KK, Chan RK, Lau CC, Tsao SW et al. (1994) Molecular cloning of differentially expressed genes in human epithelial ovarian cancer. Gynecol Oncol 52: 247-252. doi:10.1006/gyno. 1994.1040. PubMed: 8314147.

6. Matei D, Graeber TG, Baldwin RL, Karlan BY, Rao J et al. (2002) Gene expression in epithelial ovarian carcinoma. Oncogene 21: 6289-6298. doi:10.1038/sj.onc.1205785. PubMed: 12214269.

7. Quaye L, Dafou D, Ramus SJ, Song H, Gentry-Maharaj A et al. (2009) Functional complementation studies identify candidate genes and common genetic variants associated with ovarian cancer survival. Hum Mol Genet 18: 1869-1878. doi:10.1093/hmg/ddp107. PubMed: 19270026.

8. Notaridou M, Quaye L, Dafou D, Jones C, Song H et al. (2011) Common alleles in candidate susceptibility genes associated with risk and development of epithelial ovarian cancer. Int J Cancer, 128: 206374. PubMed: 20635389.

9. Schwarze SR, DePrimo SE, Grabert LM, Fu VX, Brooks JD et al. (2002) Novel pathways associated with bypassing cellular senescence in human prostate epithelial cells. J Biol Chem 277: 14877-14883. doi: 10.1074/jbc.M200373200. PubMed: 11836256

10. Schwarze SR, Fu VX, Desotelle JA, Kenowski ML, Jarrard DF (2005) The identification of senescence-specific genes during the induction of senescence in prostate cancer cells. Neoplasia 7: 816-823. doi: 10.1593/neo.05250. PubMed: 16229804.

11. Desotelle J, Truong M, Ewald J, Weeratunga P, Yang B et al. (2013) CpG Island Hypermethylation Frequently Silences FILIP1L Isoform 2 Expression in Prostate Cancer. J Urol 189: 329-335. doi:10.1016/j.juro. 2013.02.363. PubMed: 23174249

12. Ehrich M, Turner J, Gibbs P, Lipton L, Giovanneti M et al. (2008) Cytosine methylation profiling of cancer cell lines. Proc Natl Acad Sci U
S A 105: 4844-4849. doi:10.1073/pnas.0712251105. PubMed: 18353987.

13. Radpour R, Haghighi MM, Fan AX, Torbati PM, Hahn S et al. (2008) High-throughput hacking of the methylation patterns in breast cancer by in vitro transcription and thymidine-specific cleavage mass array on MALDI-TOF silico-chip. Mol Cancer Res 6: 1702-1709. doi: 10.1158/1541-7786.MCR-08-0262. PubMed: 19010818

14. Radpour R, Kohler C, Haghighi MM, Fan AX, Holzgreve W et al. (2009) Methylation profiles of 22 candidate genes in breast cancer using highthroughput MALDI-TOF mass array. Oncogene 28: 2969-2978. doi: 10.1038/onc.2009.149. PubMed: 19503099.

15. Vanaja DK, Ehrich M, Van den Boom D, Cheville JC, Karnes RJ et al. (2009) Hypermethylation of genes for diagnosis and risk stratification of prostate cancer. Cancer Invest 27: 549-560. doi: 10.1080/07357900802620794. PubMed: 19229700.

16. Khumalo T, Reusch U, Knackmuss S, Little M, Veale RB et al. (2013) Adhesion and Invasion of Breast and Oesophageal Cancer Cells Are Impeded by Anti-LRP/LR-Specific Antibody IgG1-iS18. PLOS ONE 8: e66297. doi:10.1371/journal.pone.0066297. PubMed: 23823499.

17. Mazzanti CM, Tandle A Lorang $D$, Costouros $N$, Roberts $D$ et al. (2004) Early genetic mechanisms underlying the inhibitory effects of endostatin and fumagillin on human endothelial cells. Genome Res 14: 1585-1593. doi:10.1101/gr.2552804. PubMed: 15289477.

18. Tandle AT, Mazzanti C, Alexander HR, Roberts DD, Libutti SK (2005) Endothelial monocyte activating polypeptide-II induced gene expression changes in endothelial cells. Cytokine 30: 347-358. doi: 10.1016/j.cyto.2005.01.020. PubMed: 15935955

19. Conne B, Stutz A, Vassalli JD (2000) The 3' untranslated region of messenger RNA: A molecular 'hotspot' for pathology? Nat Med 6: 637-641. doi:10.1038/76211. PubMed: 10835679.

20. Parker $\mathrm{R}$, Song $\mathrm{H}$ (2004) The enzymes and control of eukaryotic mRNA turnover. Nat Struct Mol Biol 11: 121-127. doi:10.1038/nsmb724. PubMed: 14749774

21. Nagamine $Y$, Medcalf RL, Muñoz-Cánoves $P$ (2005) Transcriptional and posttranscriptional regulation of the plasminogen activator system. Thromb Haemost 93: 661-675. PubMed: 15841310.

22. Schwanhäusser B, Busse D, Li N, Dittmar G, Schuchhardt J et al (2011) Global quantification of mammalian gene expression control. Nature 473: 337-342. doi:10.1038/nature10098. PubMed: 21593866.

23. Vogel C, Marcotte EM (2012) Insights into the regulation of protein abundance from proteomic and transcriptomic analyses. Nat Rev Genet 13: 227-232. PubMed: 22411467

24. Salari R, Wojtowicz D, Zheng J, Levens D, Pilpel Y et al. (2012) Teasing apart translational and transcriptional components of stochastic variations in eukaryotic gene expression. PLoS Comput Biol 8: e1002644. PubMed: 22956896. 